\title{
Evaluación de un programa de desnutrición infantil en el consultorio Pudahuel
}

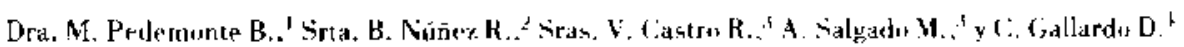

\begin{abstract}
ABSTRAC'T

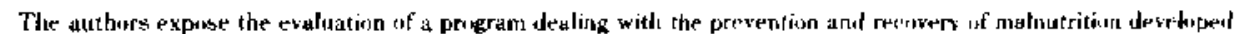

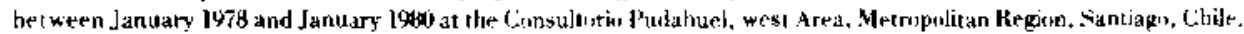

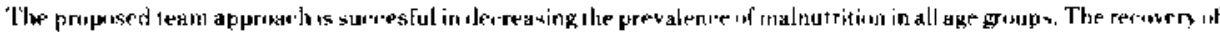

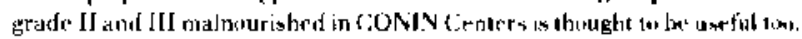

La desnutrición en la infincia constituye uni, de Ins más yrandes problemas médico-sociales de lá épo(a actual ya que las $2 / 3$ partes de la poblacion mundial la sufren en grado variable. Io que representa un serio detrimento para el desarrollo psíquico, fisico y surial del individus." Se puede afirmar que la desnutric'ión y el subdesarrollo ronstituyen en sí, un circulo viciom so. Chile no eacapa a estu realidad y las ciencias médicas y sociales iúnan sus esfuerzos dja a día prara erradirar este flagelo,

Ed consulturio Pudahuel considerandu el numero impurtante de niñus de 0 hasta 6 años controlándose en el estabtecimientw y las precarias cundiciones sociocconómicas y culturales de la población, sintis las necesidad de elahorar un programa destinaro tanto a la prevenciún fomo recuperación de la desnutricicin.

\section{OBJETIVOS}

1. Pesquisa y control de la población de altu riesgo de Pudahuel a fin de prevenir el déficil nutricional.

2. Disminución de la incidencia de la desnutririont.

3. Disminución de la prevalencia de la desnutrición.

\section{PROCEDIMIENTOS TECVICOS Y METODOLOGICOS}

A partir del año 1978 un grupo de profesionales del Consultorio de Pudahuel elaborí un prograna de stinado a la prevención y recuperación de la desmutri-

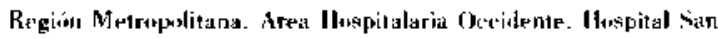
Jutan de likis.

'Módieso Cienpral Zaria Urtaria.

Entermera Jefa.

Nutricinnistan.

Haisitante sicial. rion dirigido a: nin̄os de alto riesges, niños recién macidos $y$ a desnutridas, an arciones esperificas a realizar para cada integrarte del equipo de salud.

La pvaluación del estado nutriciunal se realizi comparandu datos de baju control de niñose eutrotions y desnutridos en un periodo de dos añms tenero 1978 a enern de 1980 registrados en el Servicion de Nutrición del Consultorio de Pudahuel.

Para evaluar el estado nutritivo de los niños se lumi cono referencia las tablas de Sempé. 1:2, 1:3

Al inicio del prougrama se unificiaron eriterins con respectua:

\section{1: ALTO RIESOO}

Se consideraron tacterese de Ato Riesgu!". It

1. Viñs con baju pesu de nacimientu menus de 2.500 grr.

2. Existencia de hermanus desnutridus.

3. Antecerdentes mórbides en el ambarazo y io parts.

4. Hospit alizaciontes repetida del niñu (más de dus en un semestre).

5. Cuadrus diarréicus frecuentes.

6. Destete precoz dantes del 4. mesi.

7 . Padre yio madre alcshólica.

8. Madre enferma mental.

9. Malas rondiciemes económicas y ambientales del hogar.

10. Madre adoleseente, analfabeta.

11. Ausencia de la madre.

12. Abandono tutal del niño.

13. Cesantia del jefe del hogar.

Todos las niños que presentaron uno o más de los factores antes mencionadus fueron calificados como de alto riesso porr el equipo profesional a través de sus prestaciones de salud. ingresando al programa. donde:

a) El médico realizó examen médico y de laboratorio completo tratande patologias. que aumentaran el riesgo de desnutricion. 
b) Enfermería: mediante el contrul salud periódico y visita dumiciliaria, refurź́ a través de la educación las indicaciones entregadas e informi de las variaciones con respecto al factor de Alto Riesgo.

c) Vutrición: Aportó educación alimentaria integral de acuerdo a la edad en el momento de la entrega de los alimentos donadus pur OFASA (Vigorin. Robustin y (iranolita) ${ }^{\text {th }}$ 16 durante cinco meses.

d) Servicio Social: Delectí y controlita situación social del menor y su grupa familiar a través de la entrevista y visita dumioiliaria, realizando las gestiones currespondientes a fin de facilitar la sulueión de las problemáticas existentes. Además educó respecto a la minimización de variables que influyeron en la siruación de Alto Riesgo.

\section{Il. RECIEN NACI[YOS}

El caso del recién nacido que acudio por primera vez a control salud en enfermeria aproximadamente a los diez dias si:

1. Silo recuperio peso de nacimiento. se rinIrolín a los siele dias siguientes para evaluar el incremento y según éste se determinú la conducta a seguir.

2. No recuperis el peso de nacimiento, se envicia la madre a nutrición para recibir educ:ación alimentaria de la nudriza y sc hizo entrega de alimentos OFANA (Granulita) y, el menor fue citadn a los siete dias, rontinuando con los cuntroles periindieos por enfermería duranle dince semanas. Ciuando no hubu un aumento esperado (mínimo 140 gr por semana) se ingresoi al prograna eomo Alto Riesgo.

3. Pesó nemos de $2.000 \mathrm{gr}$ al nacer, en su primera consulta solu se tomi en cuenta el incrementw hasta esa fectha citándosele a los siete dias donde se le exigió un mínimo por semana ( 140 gr). Si no lo logroi se le introduje lecthe artificial.

III. DESNLITRIDOS

L. Sè ingresíal programa a toda nin̄on que consultí porr patologiat siendo ya un desnutrido y estando en control cem la nutricionistat. Sie trató la patolugia y se indicai seguir control con nutricinniasta y consultar médico en casu necesario.

2. Si fue la primera consulta gor déficit pondoestatural referida a médico pur enfermería u utri profesional de la salud se evaluó de acuerdo al incremento de peso-talla-edad desde el narimiento hasta la fecha de la consulta y así be comprobó si habíu desnutriciún, si era aguda o crónica y la intensidad del déficit. De aruerdo a estro:

a) Al tratarse de eutrofia limite se investigi la causal del déficit, si fue alguna patologia, se trató y se envií a nulrición como alto riesgo controlándose cada quince dias, si fue menor de seis meses de edad y. mes a mes si fue mayor de seis meses, hasta comple. tur seis meses de control, dándoseje el alta si evolucianó hacia la eutrefia, si existieron lactores socioeconomicos importantes se envií a Servicur. Sux ial.

b) Al tratarse de un desnutrido grado I se trataron painlogias concomitantes y se refiritron paralelamente a Servicio Social y Nutrición.

c) Al tratarse de un desnutrido grado II $\%$ III se envió a Servicios Social y Nutrición. para su hesspitalización. si fue posibie el mismo día en el Centro de Vutrición de Pudahuel (CONIN) si no ingresi, fue controlado por el exuipo de salud hasta su ingreso en dicho establecimiento.

\section{RESLLTADOS}

La metodología y los procedimientos técnicus antes mencionados fueron aplicados en forma ginbal a Iudos los niños de 0 hasta 6 años de edad que se inscribieron o que estaban inscritos al inicio de este estudir, enero 1978, y seguidos durante los 2 años transc'urridos de esa fecha. o sea hasta enero del presente มก๊ง.

En la tabla N." I presentamos el número total de niños bajo control a comienzo y el número total de casos al término del periodo de 2 años de observación.

Tabla 1

DGYRIBUCION DE LOS NINOS BAJO CONTREL EN EL CONBULTORIO PUPAHUEL ENERO IOTG-ENERO 1980

\begin{tabular}{|c|c|c|c|c|}
\hline & \multicolumn{2}{|c|}{ POELACION } & \multicolumn{2}{|c|}{ CONTROL } \\
\hline EOAD & \multicolumn{2}{|c|}{ ENERO-1978 } & \multicolumn{2}{|c|}{ ENFRO -1080} \\
\hline o-hosto of the & 536 & 7. $98 \%$ & 691 & $9.78 \%$ \\
\hline $\begin{array}{l}786 \text { ma. } \\
\text { mosta }\end{array}$ & 505 & 7.528 & 634 & B. $97 \%$ \\
\hline $\begin{array}{l}1 \text { ados } \\
\text { basta equar }\end{array}$ & 1.209 & $60,0 \%$ & 1.184 & $16.75 \%$ \\
\hline \multirow[t]{2}{*}{$\begin{array}{l}\text { De } 5 \text { antos } \\
\text { nosto } 6 \text { atops }\end{array}$} & 4.465 & $66.49 \%$ & 4.560 & $64.50^{\circ}$ \\
\hline & 6.715 & $99.90 \%$ & 7.060 & $99.99 \times$ \\
\hline
\end{tabular}

El estado nutricional de los $6.7 \mathbf{l n}$ nirios arriba menciunados, se visualizan en la tabla V." 2 .

Tabla 2

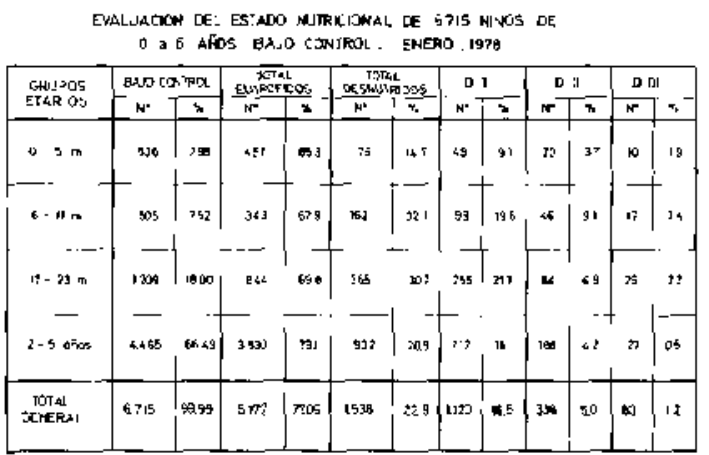

